Review began 12/02/2021 Review ended 12/03/2021 Published 12/03/2021 Exp. concern 04/07/2022

\section{Copyright 2021}

Alzahrani et al. This is an open access article distributed under the terms of the Creative Commons Attribution License CCBY 4.0 , which permits unrestricted use distribution, and reproduction in any medium, provided the original author and source are credited.

\section{Mesenteric Panniculitis: A Rare Condition in a Patient With Rheumatoid Arthritis}

Thamer S. Alzahrani ${ }^{1}$, Ali H. Alharbi ${ }^{2}$, Abdulellah I. Al Homoudi ${ }^{3}$, Arwa A. Reidi ${ }^{4}$, Farah A. Alshehri ${ }^{4}$, Nawaf F. Alsarraj ${ }^{5}$, Heba H. Milibary ${ }^{5}$, Rashed A. Alnemer ${ }^{6}$, Yousef Z. Murad ${ }^{6}$, Sarah M. Almousa ${ }^{6}$, Bashaier M. Albalawi ${ }^{7}$, Ayman J. Almalky ${ }^{8}$, Ali B. Alaithan ${ }^{9}$, Furqan H. Alawami ${ }^{9}$, Faisal Al-Hawaj 10

1. College of Medicine, Al-Baha University, Al-Baha, SAU 2. General Practice, Al-Nakheel Primary Health Care Center, Qassim, SAU 3. College of Medicine, Dar Al Uloom University, Riyadh, SAU 4. College of Medicine, Ibn Sina National College for Medical Studies, Jeddah, SAU 5. College of Medicine, King Abdulaziz University, Jeddah, SAU 6. College of Medicine, King Saud Bin Abdulaziz University for Health Sciences, Riyadh, SAU 7. College of Medicine, Tabuk University, Tabuk, SAU 8. College of Medicine, Taif University, Taif, SAU 9. College of Medicine, Vision Colleges, Riyadh, SAU 10. College of Medicine, Imam Abdulrahman Bin Faisal University, Dammam, SAU

Corresponding author: Faisal Al-Hawaj, saudidoctor2020@gmail.com

\section{Expression of Concern}

Expression of Concern date: April 07, 2022. Cite this expression of concern as Alzahrani T S, Alharbi $\mathrm{H}, \mathrm{Al}$ Homoudi I, et al. (April 07, 2022) Expression of Concern: Mesenteric Panniculitis: A Rare Condition in a Patient With Rheumatoid Arthritis. Cureus 14(4): x30. doi:10.7759/cureus.x30.

The concern relates to the provenance of this article as brought to our attention by Faisal Alhawaj, who denies authorship of this article and others published in Cureus. These articles were submitted and subsequently published purportedly as an effort coordinated by Imam Abdulrahman Bin Faisal University to ensure all medical interns publish at least one peer-reviewed article in order to qualify for enrollment in a postgraduate residency program as stipulated by The Saudi Commission for Health Specialties (SCFHS).

The journal has not been presented with enough evidence to warrant the formal retraction of these articles as both Imam Abdulrahman Bin Faisal University and The Saudi Commission for Health Specialties have failed to respond to numerous communications requesting additional information regarding these allegations. While we acknowledge that the provenance of these articles is very much in question, we cannot act until these claims have been investigated by the appropriate institutions with the results of said investigation communicated to Cureus.

The concern and this note will remain appended to the above-mentioned article until Cureus is provided with official confirmation from Imam Abdulrahman Bin Faisal University or The Saudi Commission for Health Specialties.

\begin{abstract}
Rheumatoid arthritis is a relatively common chronic inflammatory disorder affecting the synovial joints. Extra-articular manifestations of rheumatoid arthritis are not uncommon and include a wide range of cardiovascular, pulmonary, and neurological complications. Gastrointestinal involvement in rheumatoid arthritis is rare, but it can be impactful on the quality of life of patients. We present the case of a 45-year-old woman, with rheumatoid arthritis on methotrexate, who presented with a one-week history of abdominal pain. The patient visited the outpatient clinic earlier and was diagnosed with indigestion and was offered symptomatic treatment which failed to provide any improvement. Abdominal examination revealed a distended abdomen with generalized tenderness and rebound. However, the abdomen was soft with no palpable masses. Initial laboratory examination, including inflammatory parameters, was normal. The patient underwent a computed tomography scan of the abdomen with intravenous contrast which demonstrated inhomogeneous hyperdense mesenteric fat giving a mass-like lesion with a displacement of adjacent bowel loops. Such findings conferred the diagnosis of mesenteric panniculitis. The patient was started on corticosteroid therapy with oral prednisolone $40 \mathrm{mg}$ daily with close follow-up. She developed gradual improvement in her condition and the pain resolved completely after six days. The patient was reevaluated after six months from discharge and she had no recurrence. The case highlighted a rare association of mesenteric panniculitis and rheumatoid arthritis. Physicians should keep this mesenteric panniculitis in mind when they encounter patients with the autoimmune disorders who presented with abdominal symptoms.
\end{abstract}

Categories: Emergency Medicine, Radiology, General Surgery

Keywords: case report, abdominal pain, computed tomography, mesenteric panniculitis, rheumatoid arthritis 
Rheumatoid arthritis is a chronic polyarticular inflammatory disorder of unknown etiology that tends to affect small joints. The prevalence of rheumatoid arthritis reaches up to $1 \%$ of the population [1]. It is twice more common in women compared with men. While rheumatoid arthritis is primarily a disorder of synovial joints, extra-articular involvement may occur [1]. The risk factor for systemic involvement in rheumatoid arthritis include advanced age, smoking, positive rheumatoid factor or antinuclear antibody, and having human leukocyte antigen DRB1 alleles [2]. Extra-articular manifestations of rheumatoid arthritis, include renal, cardiovascular, pulmonary, and neurological manifestations [3]. However, gastrointestinal involvement in rheumatoid arthritis is rare, but it can be impactful on the quality of life of patients. It is reported that patients with rheumatoid arthritis exhibit a higher risk for having abnormal esophageal motility and reflux disease, chronic gastritis, and secondary amyloidosis [2]. Here, we present the case of a middle-aged woman with rheumatoid arthritis who developed mesenteric panniculitis, which is a rare association with few reported cases in the medical literature [3].

\section{Case Presentation}

We present the case of a 45-year-old woman who presented to the emergency department with a one-week history of abdominal pain. Her pain was located in the epigastric area and was non-radiating. She described the pain as constant and stabbing in nature. She scored it 7 out of 10 on the severity scale. The pain was not related to meals or posture. It was partially relieved by the over-the-counter analgesics. The pain was associated with nausea and vomiting. There was no history of change in bowel habits. The patient reported no history of anorexia or weight loss. She visited the outpatient clinic earlier for this pain and was diagnosed with indigestion and was offered symptomatic treatment which failed to provide any improvement.

The patient had a significant past medical history of rheumatoid arthritis. She was diagnosed at age of 34 years. The patient was using methotrexate for her disease for the last five years. She used naproxen $500 \mathrm{mg}$ almost daily for her hand pain of arthritis. She also had a history of scalp psoriasis that was well-controlled with salicylic acid shampoo. The surgical history was remarkable for tonsillectomy only. The patient was a non-smoker and never consumed alcohol. She worked as an artist but had to quit her job because she lost her hand dexterity due to rheumatoid arthritis. Her family history was remarkable for autoimmune diseases.

Upon examination, the patient appeared tired and in discomfort. Her vital signs were within the normal limits, including a temperature of $37.5^{\circ} \mathrm{C}$, pulse rate of $88 \mathrm{bpm}$, respiratory rate of $18 \mathrm{bpm}$, and blood pressure of 112/78 mmHg. Her oxygen saturation was normal. Abdominal examination revealed a distended abdomen with generalized tenderness and rebound. However, the abdomen was soft with no palpable masses. Abdominal auscultation revealed normal bowel sounds with normal intensity and frequency. Examination of other systems was non-contributory.

Initial laboratory examination included hemoglobin level of $14.2 \mathrm{~g} / \mathrm{dL}$, white cell counts of $8500 / \mu \mathrm{L}$, and platelets count of $380,000 / \mu \mathrm{L}$. She had normal levels of erythrocyte sedimentation rate and C-reactive protein. The liver and kidneys profiles were within the normal limits (Table 1). While the laboratory markers were reassuring, the treating physician was concerned about the physical examination findings and suggested further investigation with imaging studies. 


\section{Cureus}

\begin{tabular}{|c|c|c|c|}
\hline Laboratory Investigation & Unit & Result & Reference Range \\
\hline Hemoglobin & $\mathrm{g} / \mathrm{dL}$ & 14.2 & $13.0-18.0$ \\
\hline Leukocytes & $1000 / \mathrm{mL}$ & 8.5 & $4.0-11.0$ \\
\hline Platelet & $1000 / \mathrm{mL}$ & 380 & $140-450$ \\
\hline Erythrocyte Sedimentation Rate & $\mathrm{mm} / \mathrm{hr}$. & 14 & $0-20$ \\
\hline C-Reactive Protein & $\mathrm{mg} / \mathrm{dL}$ & 7.1 & $0.3-10.0$ \\
\hline Total Bilirubin & $\mathrm{mg} / \mathrm{dL}$ & 0.4 & $0.2-1.2$ \\
\hline Albumin & $\mathrm{g} / \mathrm{dL}$ & 3.9 & $3.4-5.0$ \\
\hline Alkaline Phosphatase & $\mathrm{U} / \mathrm{L}$ & 58 & $46-116$ \\
\hline Gamma-glutamyltransferase & $\mathrm{U} / \mathrm{L}$ & 24 & $15-85$ \\
\hline Alanine Transferase & $\mathrm{U} / \mathrm{L}$ & 19 & $14-63$ \\
\hline Aspartate Transferase & $\mathrm{U} / \mathrm{L}$ & 20 & $15-37$ \\
\hline Blood Urea Nitrogen & $\mathrm{mg} / \mathrm{dL}$ & 11 & $7-18$ \\
\hline Creatinine & $\mathrm{mg} / \mathrm{dL}$ & 0.8 & $0.7-1.3$ \\
\hline Sodium & $\mathrm{mEq} / \mathrm{L}$ & 138 & $136-145$ \\
\hline Potassium & $\mathrm{mEq} / \mathrm{L}$ & 3.9 & $3.5-5.1$ \\
\hline Chloride & $\mathrm{mEq} / \mathrm{L}$ & 104 & 98-107 \\
\hline
\end{tabular}

TABLE 1: Summary of the results of laboratory findings

The patient underwent a computed tomography scan of the abdomen with intravenous contrast. The scan demonstrated inhomogeneous hyperdense mesenteric fat giving a mass-like lesion with a displacement of adjacent bowel loops. It was notable that the fat surrounding the mesenteric vessels was spared giving the appearance of a fat ring sign. Multiple enlarged mesenteric and retroperitoneal lymph nodes. Such findings conferred the diagnosis of mesenteric panniculitis (Figures 1,2). 


\section{Cureus}

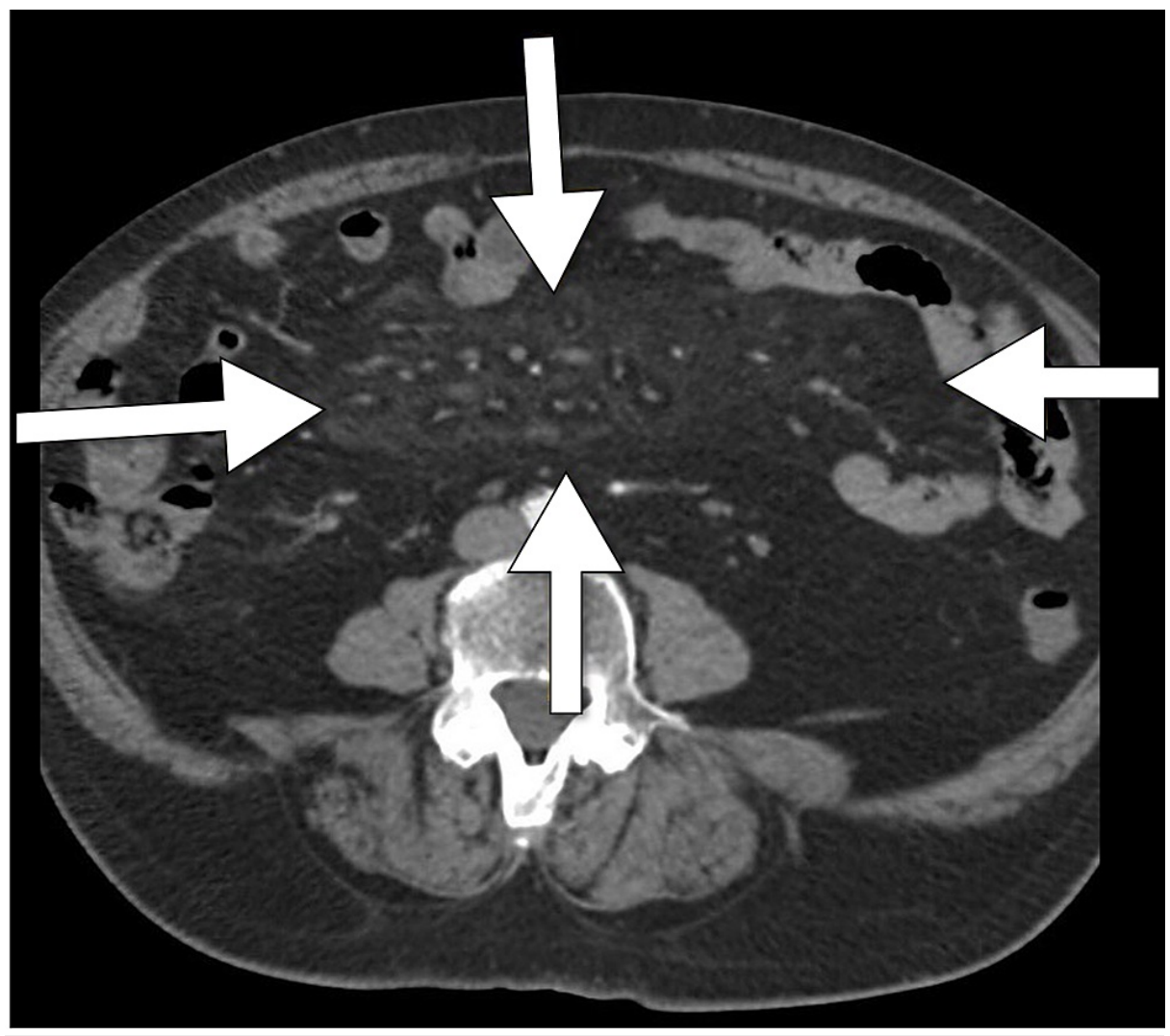

FIGURE 1: Axial CT image demonstrates inhomogeneous lesion of adipose tissue (arrows) sparing the adjacent mesenteric vessels.

CT: computed tomography 


\section{Cureus}

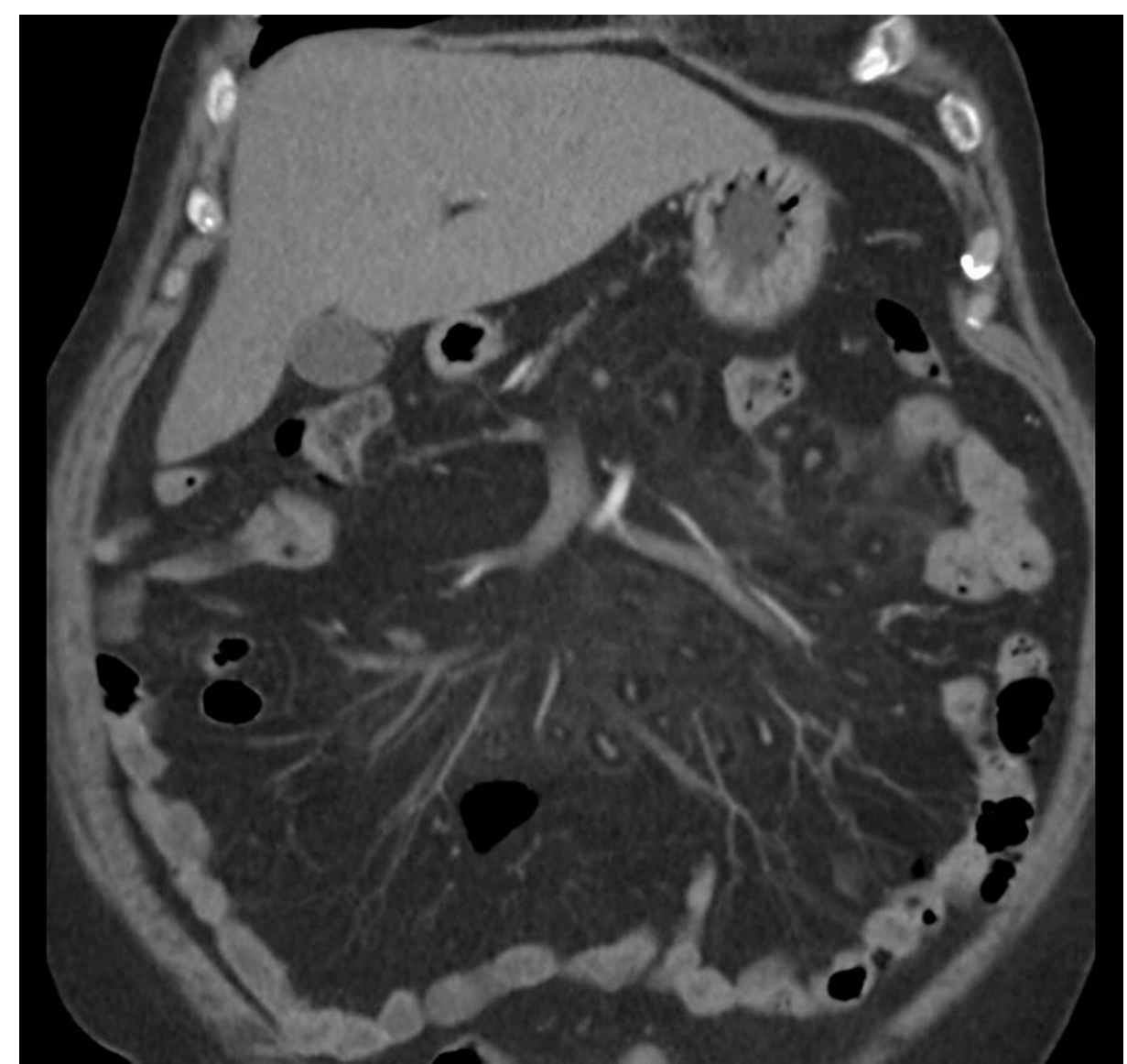

FIGURE 2: Coronal CT image demonstrates inhomogeneous hyperdense mesenteric fat sparing the mesenteric vessels giving the appearance of fat ring sign.

CT: computed tomography

The patient was started on corticosteroid therapy with oral prednisolone $40 \mathrm{mg}$ daily with close follow-up. She developed gradual improvement in her condition and the pain resolved completely after six days. She was discharged after 10 days of hospitalization. The patient had regular follow-up visits for her condition. The corticosteroid therapy was continued for two months. The patient was re-evaluated after six months from discharge and she had no recurrence or new complaints.

\section{Discussion}

We reported a rare case of mesenteric panniculitis in a middle-aged woman with rheumatoid arthritis. Mesenteric panniculitis is a rare idiopathic inflammatory condition affecting the adipose tissue [4]. It typically involves the small intestine mesentery, but the large intestine can be involved on rare occasions. The true prevalence of this condition is unknown, but a prior study indicated that it is less than $1 \%$ [5].

While the pathogenesis of mesenteric panniculitis is unclear, certain clinical factors have been shown to have a significant association with this condition. Such diseases included autoimmune disorders, infections, malignancies, and hypersensitivity reactions. In the present case, mesenteric panniculitis occurred in the setting of rheumatoid arthritis [5]. We assume that the development of mesenteric panniculitis in our patient could be related to her underlying disease directly or because of its treatment [6]. A previous study indicated that methotrexate was associated with panniculitis [7].

In contrast to the present case, mesenteric panniculitis is often asymptomatic and is detected incidentally on cross-sectional studies performed for other indications [4]. However, patients may present with a broad spectrum of manifestations, including abdominal pain and a palpable mass. The laboratory investigations, including the inflammatory parameters, lack the sensitivity and specificity to make the diagnosis of panniculitis [6]. There are many conditions that may present with a similar clinical picture to that of mesenteric panniculitis. Such conditions include a wide range of malignancies [5]. Hence, a computed tomography scan is of paramount importance to diagnose this disorder. As our patient's scan has 
demonstrated, the mesenteric panniculitis manifests radiologically as inhomogeneous adipose tissue that does not invade the surrounding bowel loops and spared the mesenteric vessels.

Regarding the management of this disorder, no indication is advised if the patient is asymptomatic as the disease resolves spontaneously. However, in the present case, the patient was given corticosteroid therapy and had a successful response. Surgical management can be attempted if the patient failed to respond to medical treatment [6].

\section{Conclusions}

The case highlighted a rare association of mesenteric panniculitis and rheumatoid arthritis. Physicians should keep this mesenteric panniculitis in mind when they encounter patients with autoimmune disorders who presented with abdominal symptoms. Cross-sectional imaging studies are crucial in making the diagnosis because of its non-specific features. Medical treatment with corticosteroid therapy is usually successful for the management of this condition.

\section{Additional Information}

\section{Disclosures}

Human subjects: Consent was obtained or waived by all participants in this study. University Institutional Review Board issued approval N/A. Case reports are waived by the institutional review board at our institution. Informed consent was obtained for the publication of this case report. Conflicts of interest: In compliance with the ICMJE uniform disclosure form, all authors declare the following: Payment/services info: All authors have declared that no financial support was received from any organization for the submitted work. Financial relationships: All authors have declared that they have no financial relationships at present or within the previous three years with any organizations that might have an interest in the submitted work. Other relationships: All authors have declared that there are no other relationships or activities that could appear to have influenced the submitted work.

\section{References}

1. Conforti A, Di Cola I, Pavlych V, et al.: Beyond the joints, the extra-articular manifestations in rheumatoid arthritis. Autoimmun Rev. 2021, 20:102735. 10.1016/j.autrev.2020.102735

2. Cojocaru M, Cojocaru IM, Silosi I, Vrabie CD, Tanasescu R: Extra-articular manifestations in rheumatoid arthritis. Maedica. 2010, 5:286-91.

3. Chao YC, Cheng TS, Chou CT: Panniculitis: an unusual cutaneous manifestation of rheumatoid arthritis . J Clin Rheumatol. 2006, 12:190-3. 10.1097/01.rhu.0000230484.04429.03

4. Gögebakan Ö, Osterhoff MA, Albrecht T: Mesenteric panniculitis (MP): a frequent coincidental CT finding of debatable clinical significance. Rofo. 2018, 190:1044-52. 10.1055/a-0633-3558

5. GU Y, TA AP, KA RO: Mesenteric panniculitis: a case report and review of the literature . Maedica. 2012, 7:344-7.

6. Tibana TK, Santos RF, Camilo DM, Marchiori E, Nunes TF: Mesenteric panniculitis in a patient with rheumatoid arthritis. Radiol Bras. 2019, 52:277-8. 10.1590/0100-3984.2017.0209

7. Al Maashari R, Hamodat MM: Methotrexate-induced panniculitis in a patient with rheumatoid arthritis . Acta Dermatovenerol Alp Pannonica Adriat. 2016, 25:79-81. 10.15570/actaapa.2016.23 